\title{
Filling Gap between Discrete and Continuous Space Flow Models in Dense Wireless Networks
}

\author{
Sina Z. Anaraki, Mehdi Kalantari \\ Electrical and Computer Engineering Department, University of Maryland College Park \\ \{anaraki, mehkalan\}@umd.edu
}

\begin{abstract}
Optimizing information flow in a dense wireless network using discrete methods can be computationally prohibitive. Instead of treating the nodes as discrete entities, these networks can be modeled as continuum of nodes providing a medium for information transport. To model information routes in continuous space, information flow vector field is defined over the geographical domain of the network. At each point of the network, the orientation of the vector field shows the direction of the flow of information, and its magnitude shows the density of information flow. Using multivariate calculus techniques in continuous domain, an information flow vector field can be found such that it minimizes a suitable cost function. Then the solution is discretized. Conventionally, a centralized method of calculating the optimal information flow in the network is suggested; however, using a centralized method to optimize information flow in a dynamic network is prohibitive. Additionally, the value of information flow vector field is needed only at the locations of nodes in the network. This poses a gap between the continuous space and discrete space models of information flow in dense wireless networks. This gap is how to calculate and apply the optimum information flow derived in continuous domain in a network with finite number of nodes. As a first step to fill this gap, a specific quadratic cost function is considered. It is proved that the the vector field that minimizes this cost function is irrotational, thus it is written as the gradient of a potential function. This potential function satisfies a Poisson Partial Differential Equation (PDE) which in conjunction with Neumann boundary condition has a unique solution up to a constant. The PDE resulted by optimization in continuous domain is first discretized and then solved in a distributed fashion. The solution requires only neighboring nodes to communicate with each other. The gradient of the resulting potential defines the routes that the traffic should be forwarded.
\end{abstract}

\section{INTRODUCTION}

A Dense Wireless Network is a network of large number of homogenous devices distributed in a geographical area to collect data about environmental conditions or events. Each device is equipped with a microcontroller, a short range radio transceiver, and a battery. Common applications of such networks include distributed systems of sensors, actuators, or monitoring and surveillance devices. Recent advancements in low power electronics and wireless communications have made it possible to manufacture such devices (which hereafter we call them nodes) in very small size and with low cost. As a result, very large scale deployment of these devices is possible in applications of interest. The information generated by each node is wirelessly transmitted to a special node with enough

This work was supported by NSF under grants CNS-0931957 and CCF0729129. energy and computational power to be further processed using network data fusion techniques. We refer to this node as the data sink or sink in short. Large networks may have several sinks. Most of the nodes that generate traffic are not in the communication range of the sinks, thus data packets should be relayed along multi-hop paths through other nodes to be delivered to data sinks.

Information flow methods model the network as a discrete set of nodes. As the number of nodes in a wireless network grows, careful behavioral analysis of a dense wireless network becomes very hard by using conventional methods that employ a discrete model in space.

A proposed method to overcome complexity issues of dense wireless network is to model the network as a continuum of nodes providing a medium for information flow. In this model, information is treated as a fluid-like entity being transported through a massively dense communication medium. The information generated by each node goes through a sequence of many small range multi-hop transmissions in the medium of nodes until it is received by the sink. This continuous space model to formulate flow of information in a wireless network was introduced in our past works [1]-[3].

In the proposed continuous space model, an information flow vector field models the transportation of nodes' traffic. This vector field has two components at each location of the network: a magnitude representing the spatial density of information that flows at that location and an orientation that gives the direction to which the traffic is forwarded. Basic flow conservation laws results in equations (similar in form to Maxwell equations) that govern information flow. Furthermore, optimizing a quadratic form of the communication cost will result in an information flow vector field that is conservative (or irrotational). As a result, the information flow vector field is the gradient of a potential function defined over the domain of the network. The potential function satisfies a Poisson Partial Differential Equation (PDE) with Neumann Boundary Condition (BC). Solution of this PDE leads to a simple mechanism for routing the traffic: each node forwards the traffic to a neighbor with least potential (steepest potential decent). In [4], it is shown that this routing method, minimizes the number of nodes required to carry a specified information density in a network area.

An important advantage of continuous space models for information flow is the use of strong analytical tools and techniques in vector calculus and partial differential equations 
for flow optimization; however, a main shortcoming of the previous works in continuous information flow modes is that the potential function that is essential for routing can only be calculated in a centralized way. All of the previous works that use continuous model for information flow assume that a central node calculates the potential as a function of space and then sends the results to all nodes. This is a significant drawback. Usefulness of continuous space models of information flow models will be questionable in absence of practical methods that calculate the potential values at the locations of nodes in a decentralized way.

The goal of this paper is to bridge the gap between continuous space models and discrete space models of information flow in massively dense wireless networks. We provide a method to compute the potential at each node in a distributed fashion such that each node computes its potential by using simple iterations. Such iterations use the potential value at a node and its neighboring nodes to find a new potential value for that node. To achieve this goal, the continuous Poisson PDE is converted to a discrete model that is valid at locations of wireless nodes using a Finite Difference Method (FDM). The discrete algorithm for finding potential function uses iterations in which each wireless node only needs to communicate with its neighboring nodes to compute and update its potential value.

The rest of this paper is organized as follows: in Section II we provide a brief summary of notations that will be used throughout the paper. Then in Section III we will introduce the earlier works on the information flow model and derive the governing PDE for the potential function. In Section IV we will use finite difference method to discretize this PDE, and obtain equations for computing its solution in a distributed fashion. A numerical example will be presented in Section V. Section VI will conclude the paper.

\section{Notations AND DEFinitions}

In this section we provide a summary of the notations and definitions that will be used throughout the paper. We will use boldface characters to represent vectors, vector fields and random variables. A position vector in $\mathbb{R}^{2}$ is denoted by $\mathbf{z}=$ $(x, y)$ or $\mathbf{z}=x \mathbf{i}+y \mathbf{j}$ in Cartesian coordinate system, where $\mathbf{i}$ and $\mathbf{j}$ are the unit vectors in the direction of $x$ and $y$ axes, respectively. In a polar coordinate system, we will use $\mathbf{z}=$ $(r, \phi)$ to specify a point that is at distance $r$ from the pole and makes the angle $\phi$ with the polar axis. The nabla operator $\nabla=\frac{\partial}{\partial x} \mathbf{i}+\frac{\partial}{\partial y} \mathbf{j}$ is used to specify the PDEs governing the information flow. The gradient of a scalar valued function of $\mathbf{z}$ is $\nabla f(\mathbf{z})=\frac{\partial}{\partial x} f(\mathbf{z}) \mathbf{i}+\frac{\partial}{\partial y} f(\mathbf{z}) \mathbf{j}$. The divergence and curl of a vector field $\mathbf{F}(\mathbf{z})=F_{x}(\mathbf{z}) \mathbf{i}+F_{y}(\mathbf{z}) \mathbf{j}$ are $\nabla \cdot \mathbf{F}=\frac{\partial}{\partial x} F_{x}+\frac{\partial}{\partial y} F_{y}$ and $\nabla \times \mathbf{F}=\left(-\frac{\partial}{\partial y} F_{x}+\frac{\partial}{\partial x} F_{y}\right) \mathbf{k}$, respectively, where $\mathbf{k}=$ $\mathbf{i} \times \mathbf{j}$ represents the unit vector in direction of $z$ axis. Note that the divergence of a vector field at a specific location is a scalar while its curl is a vector. If the vector field $\mathbf{F}$ is such that $\nabla \times \mathbf{F}(\mathbf{z})=\mathbf{0}$, then $\mathbf{F}$ is called an irrotational vector field. An important property of an irrotational vector field is that it admits a potential, i.e., it can be written as
$\mathbf{F}(\mathbf{z})=\nabla f(\mathbf{z})$, where $f$ is a scalar function. In other word, $f$ (a scalar function) determines $\mathbf{F}$ (a vector field) uniquely. The Laplacian operator is defined as $\nabla^{2}=\nabla \cdot \nabla$, which is the divergence of the gradient of a scalar function, or in Cartesian coordinates system, $\nabla^{2} f(\mathbf{z})=\frac{\partial^{2}}{\partial x^{2}} f(\mathbf{z})+\frac{\partial^{2}}{\partial y^{2}} f(\mathbf{z})$.

We assume that the network is distributed in a closed, bounded and connected region $A \subset \mathbb{R}^{2}$. We denote the boundary of this region by $\partial A$. As mentioned earlier, in a wireless sensor network, as the density of nodes increases, instead of treating nodes individually as discrete objects, we model them by a continuous space distributions and quantize this by densities. The information sources (nodes generating traffic) are distributed through the network with density $\mu_{s}(\mathbf{z})$. This means that at location $\mathbf{z}, \mu_{s}(\mathbf{z}) b p s / m^{2}$ of traffic is generated. This traffic should be routed to the sinks. We denote the density of the sinks by $\mu_{d}(\mathbf{z})$, which is interpreted like the source density, except that sinks gather the traffic generated by the sources. In the special case of a single source located at $\mathbf{z}_{0}$, the source density takes the form $\mu_{s}(\mathbf{z})=w \delta\left(\mathbf{z}-\mathbf{z}_{\mathbf{0}}\right)$ where $\delta$ represents Dirac's delta function and $w$ is the rate that the source generates information.

Since all the generated traffic in the network should be routed to the sinks, we have $\int_{A}\left(\mu_{s}(\mathbf{z})-\mu_{d}(\mathbf{z})\right) d s=0$. In order to make the equations derived in the next sections simpler, define $\rho(\mathbf{z})=\mu_{s}(\mathbf{z})-\mu_{d}(\mathbf{z})$ to be the generalized density of information sources and sinks combined. Thus we have $\int_{A} \rho(\mathbf{z}) d s=0$. The source and sink densities, $\mu_{s}$ and $\mu_{d}$ appear with opposite signs since the sources generate information traffic, whereas the sinks gather this traffic.

\section{BACKGROUND: CONTINUOUS INFORMATION FLOW MODEL}

In the most basic level, the route that information takes from a source to a sink in a wireless network can be represented by a vector field that is defined at the locations of the nodes: Each node simply forwards traffic to another node in the direction of this vector field. Minimizing a communication cost function in this scenario can be computationally prohibitive, since only discrete optimization methods can be used. However, when the density of nodes increases, this vector field becomes defined in more locations, and in the limit, it is defined over the whole region that the network is deployed. In this limit, the network is modeled by a continuum of nodes instead of a discrete set of nodes. Modeling the network as a continuum of nodes enables using vector calculus tools for optimization (e.g., Lagrangian multipliers and calculus of variations). Since in any real-world scenario the number of deployed nodes is finite, the resulted optimum vector field (or as we see later, the potential that defines it) is discretized in order to be implementable.

In our model, we denote the information flow vector field by $\mathbf{D}(\mathbf{z})$. As mentioned earlier, the direction of $\mathbf{D}(\mathbf{z})$ specifies the direction that the traffic should be forwarded by a node located at $\mathbf{z}$. We define the magnitude of $\mathbf{D}(\mathbf{z})$ to specify the density of traffic at $\mathbf{z}$, i.e., let $d \mathbf{l}$ be the differential element of length in $\mathbb{R}^{2}$, then the traffic rate crossing it is $\mathbf{D}(\mathbf{z}) \cdot d \mathbf{l}$ bps (scalar product of $d \mathbf{l}$ and $\mathbf{D}(\mathbf{z})$ ). This definition 
of information flow vector field is similar to the definition of the electric flux density in electrostatics, where its direction and magnitude specify the direction and density of electric flux passing through a surface.

Let $B \subset A$ be a closed subset of $A$. The traffic generated by information sources in $B$ should whether be gathered by a sink in $B$ or it should cross the boundary of $B$, denoted by $\partial B$. Recalling the definition of $\mathbf{D}$, we can state this by

$$
\oint_{\partial B} \mathbf{D}(\mathbf{z}) \cdot \mathbf{d} \mathbf{l}=\int_{B} \rho(\mathbf{z}) d s
$$

Equation (1) states that the total traffic crossing the boundary $B$ is equal to the net sum of all source and sink densities in that area. Note that from vector calculus, the divergence theorem states that for every continuously differentiable vector field $\mathbf{D}$ we have

$$
\oint_{\partial B} \mathbf{D}(\mathbf{z}) \cdot \mathbf{d} \mathbf{l}=\int_{B} \nabla \cdot \mathbf{D}(\mathbf{z}) d s
$$

Equations (1) and (2) hold for any closed $B \subset A$, thus we can rewrite (1) to get a point-wise version.

$$
\nabla \cdot \mathbf{D}(\mathbf{z})=\rho(\mathbf{z})
$$

This equation is similar to Gauss's flux theorem in electrostatics.

Next we need to study properties of $\mathbf{D}$ on the boundary of $A$, namely $\partial A$. All of the traffic that is generated in the network should be routed to the sinks located in $A$ and no traffic can enter or exit the boundary of network region, since we assumed that the nodes are only deployed inside of this region. In other words, D can not point outwards or inwards on the boundary, it can only be tangent to it. Thus the normal component of $\mathbf{D}(\mathbf{z})$ should be zero at the boundary. This suggests the following boundary condition (often called Neumann boundary condition) for the information flow vector field.

$$
\mathbf{D}(\mathbf{z}) \cdot \mathbf{n}=0 \quad \mathbf{z} \in \partial A
$$

where $\mathbf{n}$ is a vector perpendicular to the boundary at each $\mathbf{z}$ and it points outward.

Equations (3) and (4) do not specify $\mathbf{D}(\mathbf{z})$ uniquely, there are uncountable vector fields that satisfy both of these equations. We can define a class of cost functionals, $J$, in terms of vector field $\mathbf{D}$, and minimize it to uniquely find an optimum information flow vector field. In earlier works, we introduced a convex cost function of the form $J(\mathbf{D})=\int_{A}\|\mathbf{D}(\mathbf{z})\|^{p} d s$ for $p>1$ and investigated its properties for the different values of $p$. In [5] it is shown that $p \downarrow 1$ results in shortest path (minimum packet delay) routing while $p \uparrow \infty$ results in optimum load balancing over the network (maximum lifespan of network). For a general $p$, this cost function can be minimized numerically by Sequential Quadratic Programming (SQP) as studied in [5]. The problem with this solution is that it does not lend itself to a distributed method for computation, i.e., a central node should perform the optimization and then send the calculated information flow vector field to all of the nodes. In this paper we focus on the special case of $p=2$ (quadratic cost function), where the cost function becomes:

$$
J(\mathbf{D})=\int_{A}\|\mathbf{D}(\mathbf{z})\|^{2} d s
$$

and the optimum vector field has properties that can be exploited to devise a distributed method to calculate information flow vector field. The $p=2$ case can be viewed as a trade-off between packet delay and optimal load balancing. Furthermore, in [4] it is proved that while maintaining connectivity of network, minimizing (5) minimizes the number of nodes required to be deployed after the optimizer is discretized. Also, it is shown in [2] that the vector field that minimizes (5), $\mathbf{D}^{*}(\mathbf{z})$, has the following property:

$$
\nabla \times \mathbf{D}^{*}(\mathbf{z})=\mathbf{0}
$$

This property is essential for distributed calculation of the routes. Equation (6) suggests that the optimal information flow vector field is irrotational, thus as stated in section II we have

$$
\mathbf{D}^{*}(\mathbf{z})=-\nabla u(\mathbf{z})
$$

where $u(\mathbf{z})$ is a scalar potential function defined over $A$.

By combining equations (3) and (7) we have

$$
\nabla \cdot \nabla u(\mathbf{z})=-\rho(\mathbf{z}) \quad \mathbf{z} \in A
$$

By using the Laplacian operator notation we get

$$
\nabla^{2} u(\mathbf{z})=-\rho(\mathbf{z}) \quad \mathbf{z} \in A
$$

This equation is called a Poisson partial differential equation. The Neumann boundary condition (4) translates into:

$$
\nabla u(\mathbf{z}) \cdot \mathbf{n}=0 \quad \mathbf{z} \in \partial A
$$

thus in order to find the information flow vector field that minimizes (5) we need to solve a Poisson equation with Neumann boundary condition, which has a unique solution up to a constant.

In the next section we will develop a distributed method to calculate $u(\mathbf{z})$ that satisfies (8) and (9).

\section{A Distributed Solution For Calculating POTENTIAL FunCTION}

In this section, we will provide such a distributed method to calculate the potential function $u$ numerically at the locations of the nodes.

A classical method to solve PDEs numerically is finite difference method, where in its simplest form, the region $A$ is divided by a well defined uniform grid with spacing $h$. As depicted in Fig. 1, let us assume that a node is placed at each grid point. The second partial derivative of $u$ with respect to $x$ is approximated by the following finite difference:

$$
\frac{\partial^{2}}{\partial x^{2}} u\left(x_{1}, y_{1}\right) \simeq \frac{u\left(x_{1}-h, y_{1}\right)-2 u\left(x_{1}, y_{1}\right)+u\left(x_{1}+h, y_{1}\right)}{h^{2}}
$$




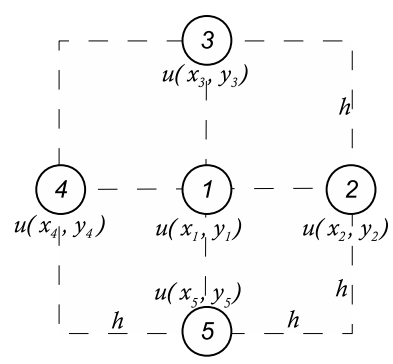

Fig. 1. Nodes $1 \ldots 5$ are placed on a grid. The potential of the nodes is calculated by (11).

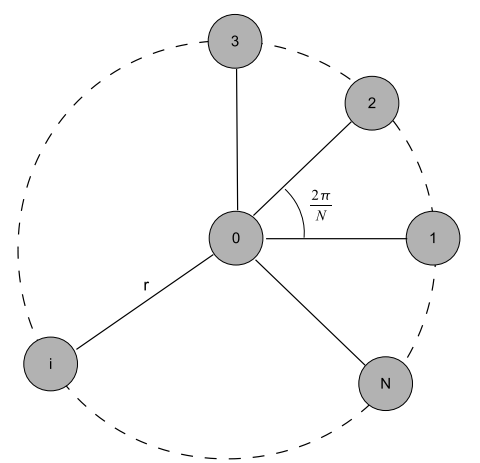

Fig. 2. Nodes $1 \cdots N$ are arranged on a circular pattern. This configuration will be used as a starting point for the situation where both $r$ and $\phi$ are randomized.

Note that $x_{1}+h=x_{2}$ and $x_{1}-h=x_{4}$. The second partial derivative of $u$ with respect to $y$ can be approximated similarly. Substituting these approximates in the Poisson equation $\frac{\partial^{2}}{\partial x^{2}} u+\frac{\partial^{2}}{\partial y^{2}} u=-\rho$ and rearranging for $u\left(x_{1}, y_{1}\right)$ we get

$$
\begin{aligned}
u\left(x_{1}, y_{1}\right) & \simeq \frac{1}{4}\left(u\left(x_{2}, y_{2}\right)+u\left(x_{3}, y_{3}\right)+u\left(x_{4}, y_{4}\right)+u\left(x_{5}, y_{5}\right)\right) \\
& +\frac{h^{2}}{4} \rho\left(x_{1}, y_{1}\right)
\end{aligned}
$$

i.e., each node should approximate its potential by averaging the potentials of its neighbors and adding a bias proportional to $\rho$. The approximation improves by increasing the number of iterations. Applying Neumann boundary conditions is simple and can be found in [6]. While this method is distributed, it can not be used in our problem, since the nodes are distributed randomly, not on a grid. We extend this method to deal with a random node deployment, where nodes are distributed uniformly and independently through the region $A$.

In the first step, assume node 0 is at position $\mathbf{z}_{\mathbf{0}}$, and nodes $1 \ldots N$ are positioned with uniform spacing around a circle with center at $\mathbf{z}_{0}$ and radius $r$. Thus, the nodes divide the circle to arcs of length $\frac{2 \pi}{N}$. Assume the pole of a polar coordinate system is placed at $\mathbf{z}_{0}$ and the polar axis is parallel to the $x$ axis. The position of nodes $1 \ldots N$ in Cartesian and polar coordinates are $\mathbf{z}_{i}=\mathbf{z}_{\mathbf{0}}+r\left(\cos \phi_{i}, \sin \phi_{i}\right)$ and $\mathbf{z}_{i}=\left(r, \phi_{i}\right)$ respectively, where $\phi_{i}=\frac{2 \pi}{N}(i-1)$. This configuration is depicted in Fig. 2.

In Cartesian coordinates, using Taylor's expansion for $u$ and keeping up to second order terms, we have

$$
\begin{aligned}
u\left(\mathbf{z}_{i}\right) \simeq & u\left(\mathbf{z}_{\mathbf{0}}\right)+\left(\mathbf{z}_{i}-\mathbf{z}_{\mathbf{0}}\right)^{T} \nabla u(\mathbf{a}) \\
& +\frac{1}{2}\left(\mathbf{z}_{i}-\mathbf{z}_{\mathbf{0}}\right)^{T} H\left(\mathbf{z}_{\mathbf{0}}\right) \frac{1}{2}\left(\mathbf{z}_{i}-\mathbf{z}_{\mathbf{0}}\right)
\end{aligned}
$$

where $H\left(\mathbf{z}_{\mathbf{0}}\right)$ is the Hessian matrix of $u$ at position $\mathbf{z}_{\mathbf{0}}$. Substituting $\mathbf{z}_{i}-\mathbf{z}_{\mathbf{0}}$ in (12) yields

$$
\begin{aligned}
u\left(\mathbf{z}_{i}\right) & \simeq u\left(\mathbf{z}_{\mathbf{0}}\right)+r\left(\frac{\partial}{\partial x} u\left(\mathbf{z}_{\mathbf{0}}\right) \cos \phi_{i}+\frac{\partial}{\partial y} u\left(\mathbf{z}_{\mathbf{0}}\right) \sin \phi_{i}\right) \\
& +\frac{1}{2} r^{2}\left(\frac{\partial^{2}}{\partial x^{2}} u\left(\mathbf{z}_{\mathbf{0}}\right) \cos ^{2} \phi_{i}+2 \frac{\partial^{2}}{\partial x \partial y} u\left(\mathbf{z}_{\mathbf{0}}\right) \sin \phi_{i} \cos \phi_{i}\right. \\
& \left.+\frac{\partial^{2}}{\partial y^{2}} u\left(\mathbf{z}_{\mathbf{0}}\right) \sin ^{2} \phi_{i}\right)
\end{aligned}
$$

We sum $u\left(\mathbf{z}_{i}\right)$ over $i=1 \ldots N$ and use identities

$$
\begin{aligned}
\sum_{i=1}^{N} \cos \phi_{i}=\sum_{i=1}^{N} \sin \phi_{i} & =0 \\
\sum_{i=1}^{N} \cos ^{2} \phi_{i}=\sum_{i=1}^{N} \sin ^{2} \phi_{i} & =\frac{N}{2} \\
\sum_{i=1}^{N} \cos \phi_{i} \sin \phi_{i} & =0
\end{aligned}
$$

which results in:

$$
\sum_{i=1}^{N} u\left(\mathbf{z}_{i}\right) \simeq N u\left(\mathbf{z}_{\mathbf{0}}\right)+N \frac{r^{2}}{4}\left(\frac{\partial^{2}}{\partial x^{2}} u\left(\mathbf{z}_{\mathbf{0}}\right)+\frac{\partial^{2}}{\partial y^{2}} u\left(\mathbf{z}_{\mathbf{0}}\right)\right)
$$

But $\frac{\partial^{2}}{\partial x^{2}} u\left(\mathbf{z}_{\mathbf{0}}\right)+\frac{\partial^{2}}{\partial y^{2}} u\left(\mathbf{z}_{\mathbf{0}}\right)=-\rho\left(\mathbf{z}_{\mathbf{0}}\right)$, thus we have

$$
u\left(\mathbf{z}_{\mathbf{0}}\right) \simeq \frac{1}{N} \sum_{i=1}^{N} u\left(\mathbf{z}_{i}\right)+\frac{r^{2}}{4} \rho\left(\mathbf{z}_{\mathbf{0}}\right)
$$

Let $N \uparrow \infty$, the Riemann sum converges to an integral, thus equation (18) would approach

$$
u\left(\mathbf{z}_{\mathbf{0}}\right) \simeq \int_{0}^{2 \pi} u(r, \phi) \frac{1}{2 \pi} d \phi+\frac{r^{2}}{4} \rho\left(\mathbf{z}_{\mathbf{0}}\right)
$$

In this equation $u(r, \phi)$ is the value of $u$ at $(r, \phi)$ in polar coordinates.

Up to here we have assumed that the nodes $1 \ldots N$ were distributed deterministically around node 0 . Now assume that all of the nodes in the network are distributed uniformly and independently in the area $A$ and that the communication range of each node is $R$. Furthermore, assume that node 0 is located at $\mathbf{z}_{0}$ inside the network far from the boundary and it has $M+$ 1 neighbors in its communication range. Theses neighboring nodes are located at $(\mathbf{r}, \boldsymbol{\Phi})$ and $\left(\mathbf{r}_{i}, \boldsymbol{\Phi}_{i}\right) i=1 \ldots M$. It is easy to show that random variables $\mathbf{r}, \mathbf{r}_{i}, \boldsymbol{\Phi}$ and $\boldsymbol{\Phi}_{i}$ are independent for $i=1 \ldots M$. Furthermore, $\boldsymbol{\Phi}$ and $\boldsymbol{\Phi}_{i} \quad i=1 \ldots M$ are uniformly distributed in the interval $[0,2 \pi)$, therefore, they are i.i.d. The random variables $\mathbf{r}, \mathbf{r}_{i} i=1 \ldots M$ are also i.i.d, but the exact expression for the distribution of $\mathbf{r}$ is not important and we will denote it by $g(r)$. Since all of the neighboring nodes are in the communication range, $g(r)$ is zero for $r>R$. 
Using (19), we will evaluate $\mathbb{E}[u(\mathbf{r}, \boldsymbol{\Phi})](\mathbb{E}[\mathbf{x}]$ denotes mathematical expectation of random variable $\mathbf{x})$ :

$$
\begin{aligned}
\mathbb{E}[u(\mathbf{r}, \boldsymbol{\Phi})] & =\int_{0}^{R} \int_{0}^{2 \pi} u(r, \phi) \frac{g(r)}{2 \pi} d r d \phi \\
& =\int_{0}^{R} g(r) d r \int_{0}^{2 \pi} u(r, \phi) \frac{1}{2 \pi} d \phi \\
& =\int_{0}^{R}\left(u\left(\mathbf{z}_{\mathbf{0}}\right)-\frac{r^{2}}{4} \rho\left(\mathbf{z}_{\mathbf{0}}\right)\right) g(r) d r \\
& =u\left(\mathbf{z}_{\mathbf{0}}\right)-\frac{\mathbb{E}\left[\mathbf{r}^{2}\right]}{4} \rho\left(\mathbf{z}_{\mathbf{0}}\right)
\end{aligned}
$$

where we have used the fact that $\int_{0}^{R} g(r) d r=1$ and $\int_{0}^{R} r^{2} g(r) d r=\mathbb{E}\left[\mathbf{r}^{2}\right]$. On the other hand, note that since $\left(\mathbf{r}_{i}, \boldsymbol{\Phi}_{i}\right)$ form an i.i.d sequence, by strong law of large numbers we have

$$
\frac{1}{M} \sum_{i=1}^{M} u\left(\mathbf{r}_{i}, \boldsymbol{\Phi}_{i}\right) \stackrel{\text { a.s. }}{\longrightarrow} \mathbb{E}[u(\mathbf{r}, \boldsymbol{\Phi})] \quad M \uparrow \infty
$$

For large $M$ we can write:

$$
u\left(\mathbf{z}_{\mathbf{0}}\right) \simeq \frac{1}{M} \sum_{i=1}^{M} u\left(\mathbf{r}_{i}, \mathbf{\Phi}_{i}\right)+\frac{\mathbb{E}\left[\mathbf{r}^{2}\right]}{4} \rho\left(\mathbf{z}_{\mathbf{0}}\right)
$$

Equation (22) states that a node located at $\mathbf{z}_{0}$ can estimate its potential simply by averaging the potentials of its neighboring nodes and adding a bias proportional to $\rho\left(\mathbf{z}_{\mathbf{0}}\right)$. Like (11), after several iterations, this estimation become more accurate. Equation (22) is well suited for distributed calculation of potential function $u$, since each node estimates its potential only based on the potential of its neighboring nodes.

In the derivation of (22), we had two important assumptions: First, $\boldsymbol{\Phi}$ is uniformly distributed over $[0,2 \pi)$, and second $\mathbf{r}$ and $\Phi$ are independent. These conditions are satisfied when node 0 is far from the boundary. However, when the distance of node 0 form the boundary is less than $R$ (the communication range) these assumptions do not hold. We call these nodes boundary nodes. Imposing Neumann boundary condition on these nodes should proceed along lines similar to those of the uniform grid case: when $M$ is large, each node on the boundary should simply average the potential of its neighboring nodes. Since the proof is lenghty, we will provide a rigorous proof in future works. The proof is based on mirror nodes on the boundary.

\section{NUMERICAL EXAMPLE}

In this section we present simulation results for the algorithm proposed for computing potential function. In the simulation setting, we scattered 1000 nodes in a unit square. The communication range of each node is 0.1 . A single source is located at $(0.1,0.5)$ and a sink is placed at $(0.9,0.5)$. Other nodes act as relays to pass the traffic from source to sink. In order to compute the potential function $u$, each node first finds the neighboring nodes in its communication range and then uses (22) to calculate its potential. This process is

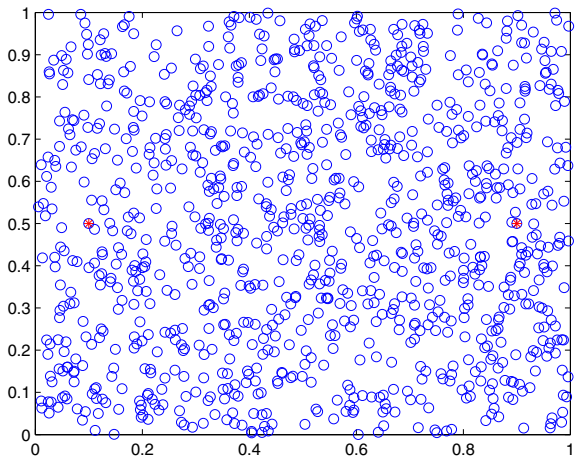

Fig. 3. The position of nodes in the simulated network. The nodes are distributed uniformly and independently.

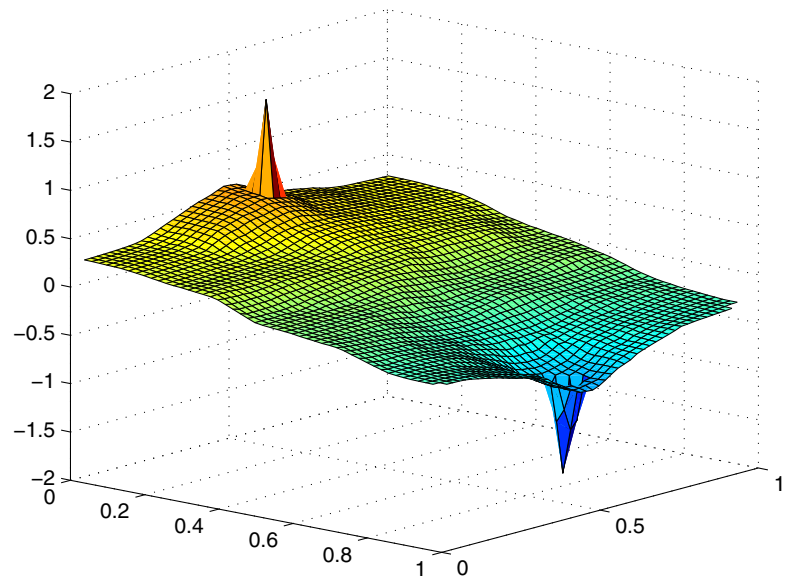

Fig. 4. The computed potentials after 10000 iterations.

carried on for 10000 iterations to increase the accuracy of the potential. Note that after much smaller number of iterations the information flow path converge and remain almost unchanged. Figure 3 depicts the placement of the nodes. The nodes are distributed uniformly and independently in the area. Figure 4 depicts the calculated potential, where the potential is interpolated on a grid and then plotted. Figure 5 shows the potential function when calculated by MATLAB assempde function, where the potentials are calculated by finite element method on a uniform grid. It is observable that the two graph follow the same pattern: a peak at the source and a smooth slope to the sink. Note that the source causes the potential of neighboring nodes to increase while the sink causes this potential to decrease. After calculating the potentials, when a node needs to forward a packet, it searches among its neighbors and sends its packet to the nearest neighbor with least potential. The slope from source to sink in the potential function guarantees that the packets will be delivered to the sink. 


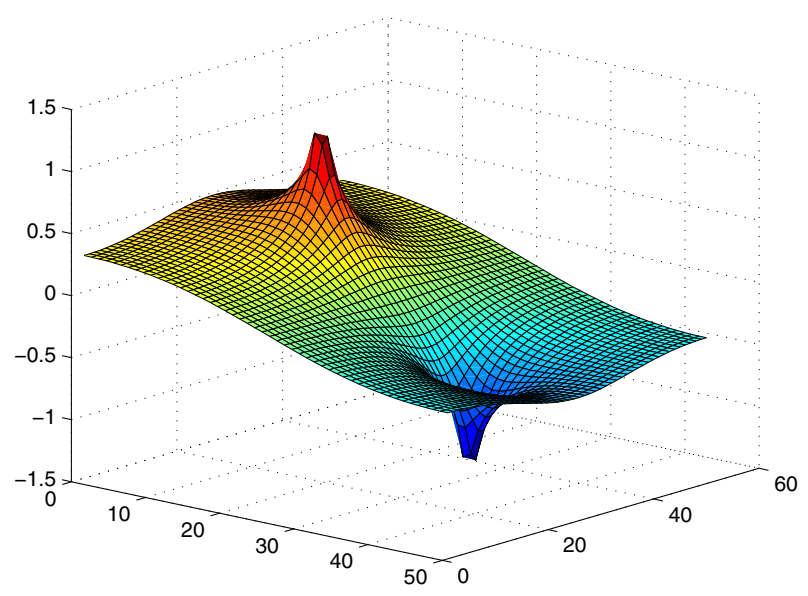

Fig. 5. The potential computed using MATLAB assempde function.

\section{CONCLUSion}

In this paper we proposed a distributed method to bridge the gap between continuous and discrete space models for information flow in wireless networks. We used the flow information vector field to model a dense wireless network. In the model optimization of the information flow paths lead to a Poisson PDE for a potential function. This potential function played a crucial role in calculating the optimal flow. In all previous works, it was assumed that this potential function is calculated in a central node and the result is sent back to the nodes. In this paper, initially, we studied the classical method for solving PDEs numerically based on finite element method. Although this method is powerful for numerically solving PDEs on a uniform grid, it can not be used directly in our model, since the nodes are distributed randomly. We generalized the finite difference method to accommodate with the random node distribution. In our proposed method, the potential of each node is computed by only communicating with the neighboring nodes: Each node updates its own potential by simply averaging the potential of its neighboring nodes and adding a bias proportional to its traffic activity. When this potential is computed, the traffic is forwarded from high potential nodes to the low potential ones. This solution diminishes the need of central node (which in many applications is a single point of failure and reduces the reliability of the network) to calculate the potentials.

\section{REFERENCES}

[1] M. Kalantari and M. Shayman, "Routing in wireless ad hoc networks by analogy to electrostatic theory," in 2004 IEEE International Conference on Communications, vol. 7, 2004.

[2] — "Energy efficient routing in wireless sensor networks," in Proc. Conference on Information Sciences and Systems. Citeseer, 2004.

[3] _ - "Routing in multi-commodity sensor networks based on partial differential equations," in Information Sciences and Systems, 2006 40th Annual Conference on, 2006, pp. 402-406.
[4] S. Toumpis and L. Tassiulas, "Optimal deployment of large wireless sensor networks," IEEE Transactions on Information Theory, vol. 52, no. 7, pp. 2935-2953, 2006.

[5] M. Kalantari, M. Haghpanahi, and M. Shayman, "A p-norm Flow Optimization Problem in Dense Wireless Sensor Networks," Proc. International Conference on Computer Communications, 2008.

[6] J. Hoffman, Numerical methods for engineers and scientists. CRC, 2001. 